\title{
Avaliação técnica de um transportador pneumático de grãos por aspiração
}

\author{
Technical evaluation of a suction pneumatic grain conveyor
}

\author{
Luís Antônio Bortolaia ${ }^{\mathrm{I}}$ Pedro Luís Andrighetto ${ }^{\mathrm{II}}$ Mateus Benatti ${ }^{\mathrm{III}}$
}

\section{- NOTA-}

\section{RESUMO}

Este trabalho apresenta os resultados da avaliação técnica do protótipo do transportador pneumático de grãos por aspiração ASP 200. O equipamento foi construído para uso em instalações armazenadoras agrícolas, na limpeza de pés de elevadores, moegas e renovação do ar em galerias de silos. Os testes avaliaram características mecânicas do equipamento, descargas de material e ar, perdas de carga, vedação do equipamento, eficiência dos captores e o diâmetro das mangueiras. Os resultados subsidiaram diversas recomendações para melhoria no projeto do equipamento, que aumentarão a sua eficiência operacional. Embora o transporte pneumático seja considerado uma ciência experimental, os autores notaram a falta de procedimentos, parâmetros e orientações para testes e comparações na literatura, que são fundamentais para o desenvolvimento do projeto desses transportadores.

Palavras-chave: engenharia agrícola, máquinas agrícolas, projeto de máquinas agrícolas.

\section{ABSTRACT}

This research shows the results that were obtained from technical evaluation of ASP 200 prototype that is a suction pneumatic grain conveyor. This equipment was built to use in agricultural storage facilities to clean bucket elevator feet, loading hoppers and air renovation in silo galleries. The tests have evaluated mechanical characteristics of conveyor, air and material discharges, pressure losses, air infiltration, intake nozzle efficiency and piping diameters. The results have allowed several recommendations to improve equipment design that will increase its operational efficiency. Although pneumatic conveying is considered an experimental science, the authors have perceived the lack of procedures, parameters and orientations to conduct tests and comparisons in literature, that are fundamental to develop a design of this conveyor type.

Key words: agricultural engineering, agricultural machines, agricultural machine design.

Os transportadores pneumáticos são tradicionalmente aplicados para a movimentação de grandes massas de grãos em estruturas portuárias (NONNENMACHER, 1983). Nas instalações armazenadoras agrícolas brasileiras, são também aplicados para coleta e transporte pneumático de amostras de grãos para análise em laboratório (WEBER, 2005). O transporte dos grãos entre os equipamentos é realizado, predominantemente, através de transportadores mecânicos, como elevadores de canecas e correias transportadoras, entre outros.

Um dos problemas mais sérios na operação dos transportadores mecânicos ocorre quando há falta de energia elétrica. A parada brusca de um elevador de canecas causa o seu embuchamento pelo acúmulo de grãos no pé do elevador, situado a vários metros de profundidade. Para recolocá-lo em funcionamento, é necessária a remoção desses grãos, feita atualmente de forma manual, em espaço confinado no fundo do poço do elevador. Além da exposição dos operários aos gases tóxicos existentes (WEBER, 2005), causa transtornos pela parada da instalação por um longo tempo durante o recebimento de grãos no período de colheita.

'Departamento de Tecnologia, Campus Panambi, Universidade Regional do Noroeste do Estado do Rio Grande do Sul (UNIJUí). Av. Rudi Franke, 540, 98280-000, Panambi, RS, Brasil. E-mail: luis.bortolaia@unijui.edu.br. Autor para correspondência.

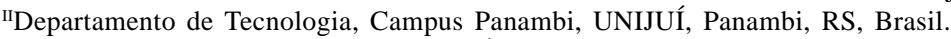

IIICurso de Engenharia Mecânica, UNIJUÍ, Panambi, RS, Brasil. 
Buscando solucionar de forma inovadora e rápida este problema, a empresa Cereall (Panambi, RS, Brasil) desenvolveu o protótipo de um equipamento transportador pneumático por aspiração, o modelo ASP 200, que também pode ser usado na limpeza de moegas e na renovação de ar em galerias de silos através do insuflamento de ar antes da realização de inspeções de rotina.

O equipamento foi desenvolvido seguindo um processo informal, adotado por diversas empresas de pequeno porte do setor industrial de máquinas agrícolas brasileiro, situação já diagnosticada por ROMANO (2003). Esta realidade ocasiona o desenvolvimento de equipamentos agrícolas com diversas imperfeições, ineficiências e, como verificado neste caso, desempenho operacional aquém do esperado. Tais máquinas muitas vezes precisam ser testadas e avaliadas para orientar fabricantes e usuários quanto às melhorias necessárias em seu projeto. ALONÇO et al. (2006) evidenciaram a importância da realização, da discussão e do relato destes testes para o desenvolvimento dos equipamentos. NONNENMACHER (1994) considera o transporte pneumático uma ciência experimental, pois, mesmo aplicando-se os fundamentos teóricos básicos no seu projeto, o desempenho do equipamento resultante pode diferir muito do projetado. Isto ressalta a importância de testar estes equipamentos para avaliar e orientar as correções necessárias. Na literatura, há poucos trabalhos relatando testes de funcionamento, metodologias de avaliação, variáveis a serem aferidas e padrões para comparação em transportadores pneumáticos. Visando a contribuir para o desenvolvimento de transportadores pneumáticos para uso com produtos agrícolas, este trabalho relata os principais procedimentos, métodos, resultados e recomendações decorrentes da avaliação do transportador pneumático ASP 200.

O ASP 200 é um transportador pneumático em aspiração, composto por uma mangueira flexível com 15m de comprimento e diâmetro 50mm (2”), e um ciclone em plástico com volume aproximado de 200 litros, que também é o reservatório dos sólidos aspirados. A descarga do produto acumulado no reservatório é feita manualmente pelo deslocamento de uma chapa metálica em sua extremidade inferior. $\mathrm{O}$ ar é succionado do interior do ciclone por uma mangueira flexível com diâmetro 75mm (3”) conectada à aspiração de um compressor radial, acionado por um motor elétrico trifásico de dois pólos com potência de $7,5 \mathrm{cv}$. O equipamento é montado sobre um chassi metálico com pneus que permitem a sua fácil movimentação (Figura 1a).
O equipamento foi testado nas instalações da UNIJUÍ, em Panambi, RS, e foram avaliados os seguintes itens: falhas mecânicas no equipamento; descarga de material por hora $\left(\mathrm{G}_{\mathrm{m}}\right)$; velocidade do ar de transporte $\left(\mathrm{v}_{\mathrm{ar}}\right)$; descarga de ar por hora $\left(\mathrm{G}_{\mathrm{ar}}\right)$; perdas de carga na mangueira de 2 " $\left(\Delta \mathrm{p}_{2}\right)$ e no ciclone $\left(\Delta \mathrm{p}_{\mathrm{C}}\right)$; vedação do equipamento; eficiência dos bocais de captação de produto (Figura 1b) e o diâmetro da mangueira de aspiração.

A presença de falhas mecânicas no equipamento foi verificada através de inspeção visual durante os testes. A descarga de material $\mathrm{Gm}$ foi medida a partir do volume transportado durante um intervalo de tempo $\left(\mathrm{m}^{3} \mathrm{~h}^{-1}\right)$, convertido para $\mathrm{kg} \mathrm{h}^{-1}$ em função do peso específico aparente do material. Os testes foram realizados com milho $\left(\gamma_{\text {milho }}=717,69 \mathrm{~kg} \mathrm{~m}^{-3}\right)$. A velocidade do ar foi medida através de tubos de Pitot instalados nas mangueiras de 2" e 3”, usando-se manômetros do tipo U. A posição radial dos tubos de Pitot foi variada conforme recomendação da norma AMCA210-85(1986). A descarga de ar $\mathrm{G}_{\mathrm{ar}}\left(\mathrm{kg} \mathrm{h}^{-1}\right)$ foi obtida multiplicandose a velocidade medida pela área da seção transversal de cada mangueira e a massa específica do ar na temperatura de teste $\left(33^{\circ} \mathrm{C}\right)$. Manômetros em $\mathrm{U}$ foram usados para medir as perdas de carga $\Delta \mathrm{p}_{2}$ e $\Delta \mathrm{p}_{C}$ através de tomadas de pressão estática situadas junto à extremidade de sucção, antes e após o ciclone. A vedação do equipamento foi avaliada calculando-se a diferença entre as vazões de ar medidas nas duas mangueiras e o equipamento aspirando apenas ar, sem transporte de material. A eficiência dos captores foi avaliada através da comparação das vazões mássicas de produto transportado. O diâmetro da mangueira de aspiração foi avaliado comparando-se as velocidades medidas com as recomendadas para o transporte de milho e a análise das perdas de carga medidas.

No teste de vedação, foram medidas as vazões de ar $\mathrm{Q}_{\mathrm{ar}}=4,60 \mathrm{~m}^{3} \mathrm{~min}^{-1}$ na mangueira de 2" e $\mathrm{Q}_{\mathrm{ar}}=7,69 \mathrm{~m}^{3} \mathrm{~min}^{-1}$ na mangueira de 3”. A diferença $\left(3,09 \mathrm{~m}^{3} \mathrm{~min}^{-1}\right)$ é a entrada falsa de ar, causada principalmente por vedação ineficiente no ciclone, que necessitou de vedação suplementar. A colocação de restrições adicionais na mangueira de sucção com o equipamento vedado provocou aumento no nível de vácuo, seguido de deformações no corpo do ciclone (Figura 1c) e na mangueira de 3" (Figura 1d). Houve ruptura do ciclone (Figura 1c), que necessitou ser reparado para continuação dos testes.

O transporte de milho foi testado sem captor (Teste A), medindo-se $\mathrm{Q}_{\mathrm{ar}}=3,06 \mathrm{~m}^{3} \mathrm{~min}^{-1}$ na mangueira de 2" $\left(\mathrm{v}_{\mathrm{ar}}=26,09 \mathrm{~m} \mathrm{~s}^{-1}\right)$ e perdas de carga $\Delta \mathrm{pC}=124 \mathrm{mmCA}$ e $\Delta \mathrm{p}_{2}=1.250 \mathrm{mmCA}$. O valor médio da descarga de material foi $G_{m}=1.077,02 \mathrm{~kg} \mathrm{~h}^{-1}$. Nos testes com o bocal 


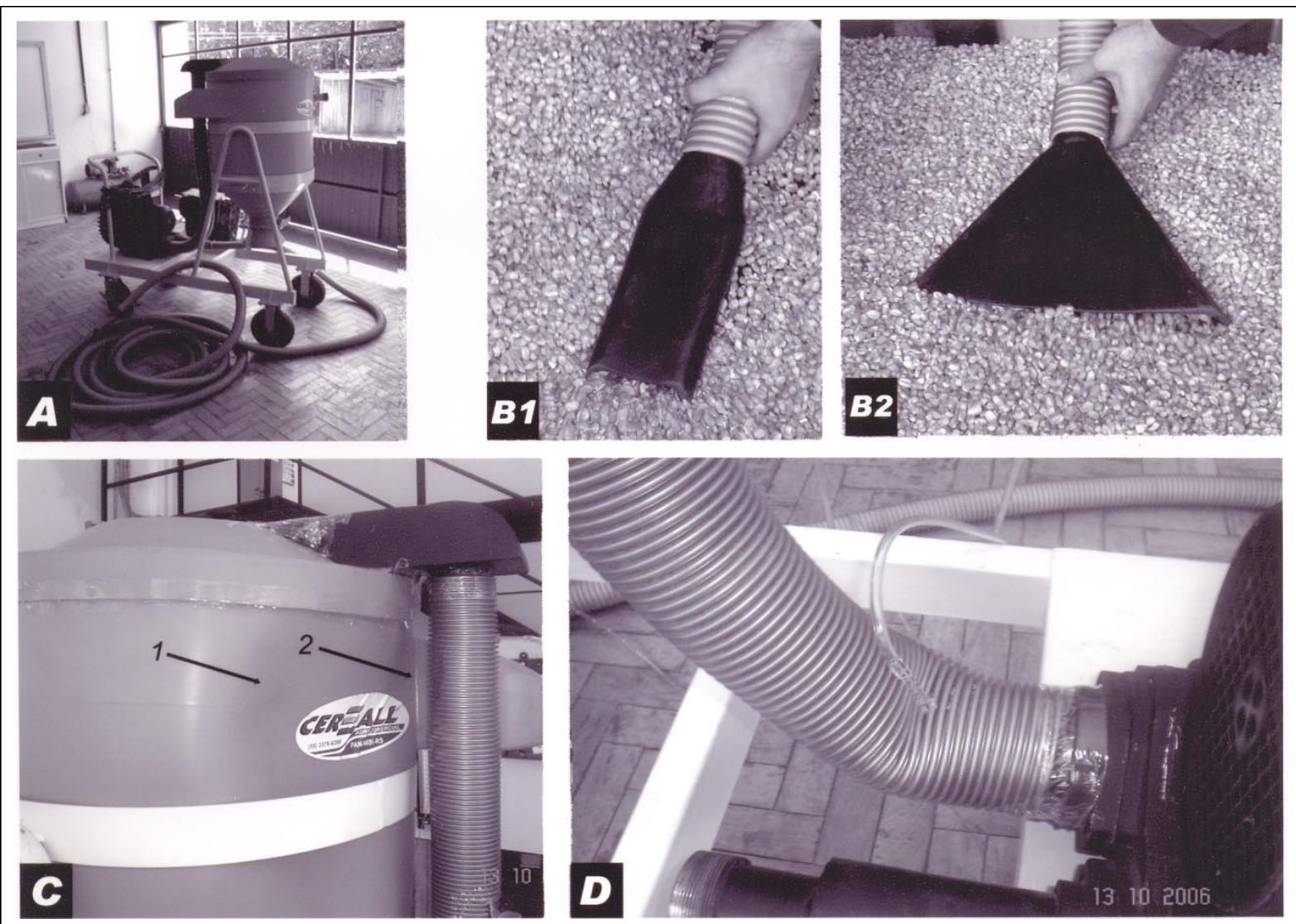

Figura 1 - Transportador pneumático ASP 200: vista geral do equipamento (A); bocais captores pequeno (B1) e grande (B2) acoplados à mangueira de sucção; deformação (C1) e ruptura (C2) no ciclone e deformação na mangueira de 3 ” conectada à aspiração do compressor radial (D).

pequeno (Teste $B$ ), mediram-se $\mathrm{Q}_{\mathrm{ar}}=3,69 \mathrm{~m}^{3} \mathrm{~min}^{-1}$ na mangueira de 2" $\left(\mathrm{v}_{\mathrm{ar}}=31,31 \mathrm{~m} \mathrm{~s}^{-1}\right)$ e perdas de carga $\Delta \mathrm{p}_{\mathrm{C}}=118 \mathrm{mmCA}$ e $\Delta \mathrm{p}_{2}=1173 \mathrm{mmCA}$. O valor médio de $\mathrm{G}_{\mathrm{m}}$ foi de $854,60 \mathrm{~kg} \mathrm{~h}^{-1}$. Para os testes com o bocal grande (Teste $C$ ), a vazão de ar foi $\mathrm{Q}_{\mathrm{ar}}=3,41 \mathrm{~m}^{3} \mathrm{~min}^{-1}$ na mangueira de 2 " $\left(\mathrm{v}_{\mathrm{ar}}=28,94 \mathrm{~m} \mathrm{~s}^{-1}\right)$ e perdas de carga $\Delta \mathrm{p}_{\mathrm{C}}=142 \mathrm{mmCA}$ e $\Delta \mathrm{p}_{2}=1.285 \mathrm{mmCA}$. O valor médio de $\mathrm{G}_{\mathrm{m}}$ foi de $833,42 \mathrm{~kg}$ $h^{-1}$. Nos testes, foi verificada a presença de eletricidade estática, causada pelo atrito provocado pelo movimento dos grãos. O operador do equipamento ficou sujeito a choques elétricos.

As perdas de carga medidas $\Delta \mathrm{p}_{2}$ e $\Delta \mathrm{p}_{\mathrm{C}}$ foram comparadas com as calculadas segundo o Roteiro Básico para Dimensionamento apresentado em NONNENMACHER (1994). Os valores calculados para $\Delta \mathrm{p}_{2}$ são: 1.283,2mmCA(Teste A), 1.382,6mmCA(Teste B) e 1.213,4mmCA (Teste C). Para $\Delta p_{C}$, foram obtidos valores de 124,5mmCA, 147,1mmCA (Teste B) e 127,4mmCA (Teste C). Os resultados calculados têm boa concordância com os valores experimentais, validando ambos.
A relação em peso $r_{p}$ é definida como a relação entre a descarga de material $\left(\mathrm{kg} \mathrm{h}^{-1}\right)$ e a descarga de $\operatorname{ar}\left(\mathrm{kg} \mathrm{h}^{-1}\right)$. Para o Teste A, obteve-se $\mathrm{r}_{\mathrm{p}}=4,83$. Para os Testes B e C, os valores foram de 3,35 e 3,54, respectivamente. O valor recomendado para o transporte pneumático de milho é $\mathrm{r}_{\mathrm{p}}=5,46$ (NONNENMACHER, 1983), indicando que o equipamento deveria transportar uma quantidade maior de produto usando a mesma quantidade de ar. Os resultados pioram com a utilização dos captores, quando o equipamento embucha com maior facilidade.

A velocidade do ar recomendada para o transporte de milho é de 32,59 $\mathrm{m} \mathrm{s}^{-1}$ (NONNENMACHER, 1994). Este valor é superior aos valores experimentais apurados, em função das perdas de carga elevadas e da falta de vedação no ciclone. O uso de velocidade menor que a recomendada afeta o desempenho do transportador, reduzindo a sua eficiência. Por outro lado, o uso de uma velocidade maior que a recomendada causa danos ao produto e também reduz a eficiência do equipamento, devido ao aumento da perda de carga

Ciência Rural, v.38, n.2, mar-abr, 2008. 
(GOMES et al., 2006). A utilização dos captores testados diminui a sucção do produto, o que reduz a perda de carga na mangueira de sucção, mudando o ponto de operação do compressor, o que é comprovado pelo aumento na descarga e velocidade do ar medidas.

Para operações de limpeza, a descarga recomendada de produto para transporte pneumático com um único operador situa-se em torno de $2.000 \mathrm{~kg} \mathrm{~h}^{-1}$ (SILVA, 2005), valor bem superior ao medido no equipamento testado. Aplicando-se o Roteiro Básico para Dimensionamento (NONNENMACHER, 1994), verifica-se que a alteração do diâmetro da mangueira de sucção para 2,5” (63,5mm) torna possível atingir esta descarga de milho, com perda de carga total $\left(\Delta \mathrm{p}_{2}\right.$ $+\Delta \mathrm{p}_{\mathrm{C}}$ ) calculada de $1.400 \mathrm{mmCA}, \mathrm{Q}_{\mathrm{ar}}=5,24 \mathrm{~m}^{3} \mathrm{~min}^{-1} \mathrm{e}$ potência de acionamento de $3,2 \mathrm{cv}$, que podem ser atingidos por este equipamento. Calculando-se o efeito de uma altura vertical de sucção de $6,0 \mathrm{~m}$, correspondente ao uso do equipamento em uma situação de desembuchamento de pé de elevador, obteve-se perda de carga total $=1.711 \mathrm{mmCA}$ e potência de acionamento de 3,9cv, considerando-se a mangueira de sucção de 2,5”. Simulando-se o transporte pneumático de soja nas situações acima, verifica-se que ele exigirá uma descarga de ar cerca de 5\% maior e uma potência de acionamento cerca de $13 \%$ maior. A perda de carga simulada será cerca de 7\% maior.

Os resultados dos testes e os novos valores calculados foram importantes para subsidiar a melhoria do projeto do equipamento. Foi recomendado ao fabricante a revisão dos seguintes itens: redimensionar os captores, que deverão incluir tomadas de ar secundária reguláveis, fundamentais para otimizar a sucção de material; alterar o material de construção do ciclone, que deverá ser em chapa de aço carbono, para eliminar deformações e infiltrações de ar; o ciclone deverá ser aterrado e as mangueiras flexíveis providas de fios de cobre em sua parede para eliminar a eletricidade estática; deverá ser usada tubulação rígida ligando o ciclone ao compressor e a mangueira de sucção deverá ter o seu diâmetro aumentado para 2,5”.

Os resultados encontrados permitem afirmar que a realização de testes para avaliação técnica de transportadores pneumáticos é fundamental para o desenvolvimento de seu projeto fluidodinâmico.
Embora o transporte pneumático seja considerado uma ciência experimental, estes equipamentos são normalmente avaliados apenas pela descarga de produto que são capazes de fornecer, dando pouca ênfase a variáveis como velocidades, vazões de ar e perdas de carga. A avaliação destes parâmetros é um procedimento experimental muito importante, embora trabalhoso, e que encontra poucos parâmetros para comparação e orientação na literatura. Como continuidade, pretende-se desenvolver uma metodologia de avaliação de transportadores pneumáticos usando instrumentação de baixo custo, relacionando os itens dos equipamentos que devem ser avaliados e indicando parâmetros para comparação.

\section{REFERÊNCIAS}

ALONÇO, A.S. et al. Avaliação técnica de uma máquina para a correção de microrrelevo do solo de áreas destinadas ao cultivo de arroz irrigado. Ciência Rural, v.36, p.16431646, 2006

AIR MOVEMENT AND CONTROL ASSOCIATION. AMCA 210-85: laboratory methods of testing fans for rating. Estados Unidos: Air Movement and Control Association, 1986. 60p.

GOMES, L.M. et al. Análise experimental de velocidade de captura em sistemas de transporte pneumático. In: CONGRESSO NACIONAL DE ENGENHARIA MECÂNICA, 2006, Recife, PE. Anais... Rio de Janeiro: Associação Brasileira de Engenharia e Ciências Mecânicas, 2006. CD-ROM.

NONNENMACHER, H. Projeto, construção e teste de um transportador pneumático de grãos. 1983. 168f. Dissertação (Mestrado em Engenharia Rural) - Curso de Pós-graduação em Engenharia Rural, Universidade Federal de Santa Maria.

NONNENMACHER, H. Curso de transporte pneumático. Santa Maria: FATEC, 1994. 71p.

ROMANO, L.N. Modelo de referência para o processo de desenvolvimento de máquinas agrícolas. 2003. $285 \mathrm{f}$. Tese (Doutorado em Engenharia Mecânica) - Programa de Pós-graduação em Engenharia Mecânica, Universidade Federal de Santa Catarina.

SILVA, D.R. Tranporte pneumático. Tecnologia, projetos e aplicações na indústria e nos serviços. São Paulo: Artliber, 2005. 172p.

WEBER, E.A. Excelência em beneficiamento e armazenagem de grãos. Canoas: Salles, 2005. 586p. 